Check for updates

Cite this: RSC Adv., 2019, 9, 18008

\title{
Direct synthesis of covalent triazine-based frameworks (CTFs) through aromatic nucleophilic substitution reactions $\uparrow$
}

\author{
Tao Chen, (D) ab Wen-Qian Li, ${ }^{\text {ab }}$ Wei-Bo Hu, (iD *a Wen-Jing Hu, ${ }^{a}$ Yahu A. Liu, (D) c \\ Hui Yang (iD *ad and Ke Wen (D) *ad
}

Received 19th April 2019 Accepted 4th June 2019

DOI: $10.1039 / c 9 r a 02934 f$

rsc.li/rsc-advances

\begin{abstract}
Despite extensive efforts, only three main strategies have been developed to synthesize covalent triazinebased frameworks (CTFs) thus far. We report herein a totally new synthetic strategy which allows $\mathrm{C}-\mathrm{C}$ bonds in the CTFs to be formed through aromatic nucleophilic substitution reactions. The assynthesized CTF-1 and CTF-2 exhibited photocatalytic water splitting activity comparable to the CTFs made using ionothermal or Brønsted acid-catalyzed polymerization. Interestingly, CTF-2 distinguished itself by its two-photon fluorescence (emission at $\sim 530 \mathrm{~nm}$ under irradiation at either $400 \mathrm{~nm}$ or $800 \mathrm{~nm}$ ).
\end{abstract}

Covalent organic networks (CONs), usually porous and physicochemically stable light-weight materials, ${ }^{1}$ can accommodate, interact with, and discriminate molecules, which provides CONs with many potential applications in catalysis, ${ }^{1 i, 2}$ gas separation/storage, ${ }^{1 b, 1 i, 2 a, 3}$ chemical sensing, ${ }^{4}$ drug delivery, ${ }^{5}$ and photovoltaic light harvesting. ${ }^{1 b, 2 a, 2 b, 6}$ As a subclass of CONs, covalent triazine-based frameworks (CTFs) have attracted researchers' increasing attention worldwide. CTFs represent a class of functionalized polymers based on the trimerization and subsequent oligomerization of aromatic dinitrile monomers in the presence of a catalyst. In brief, there have been three main strategies to synthesize CTFs from aromatic dinitrile monomers: (1) polymerization ${ }^{7}$ of aromatic dinitrile monomers mediated by $\mathrm{ZnCl}_{2}$ (ionothermal process), or catalyzed by strong Brønsted acid; (2) Friedel-Crafts reaction using 2,4,6-trichloro1,3,5-triazine (cyanuric chloride); ${ }^{8}$ and (3) nickel-catalyzed Yamamoto-type Ullmann cross-coupling of 2,4,6-tris-(4-bromophenyl)-[1,3,5]triazine. ${ }^{9}$ While the $\mathrm{ZnCl}_{2}$-mediated ionothermal reaction is still the most widely used method, the high reaction temperature $\left(400{ }^{\circ} \mathrm{C}\right)$ and long reaction time $(40 \mathrm{~h})$ limited its practical application. Although variations of the method to reduce the reaction time have been reported, the difficulty of completely removing residual $\mathrm{ZnCl}_{2}$ remained as another obstacle. $^{\text {.b }}$ The Brønsted acid method allows the polymerization to occur at ambient temperature, but it can only be applied to

${ }^{a}$ Shanghai Advanced Research Institute, Chinese Academy of Sciences, Shanghai, 201210, P. R. China. E-mail: huwb@sariac.cn; yangh@sari.ac.cn; wenk@sari.ac.cn ${ }^{b}$ University of Chinese Academy of Sciences, Beijing, 100049, P. R. China

'Medicinal Chemistry, ChemBridge Research Laboratories, San Diego, CA 92127, USA ${ }^{d}$ School of Physical Science and Technology, Shanghai Tech University, Shanghai 201210, P. R. China

$\dagger$ Electronic supplementary information (ESI) available. See DOI: 10.1039/c9ra02934f certain kinds of aromatic dinitrile monomers. An important environmental issue should not be overlooked in using aromatic dinitrile monomers, especially at large scale, as the dinitrile monomers were often made using over stoichiometric amounts of toxic cyanides, which is of neither economical nor ecological interest. Friedel-Crafts alkylation could work even without any addition of solvent, and is time-efficient, but residues of activating and/or bulking agents need to be removed and recovered upon framework formation. In addition, this approach is only limited to rigid and sterically demanding building blocks. Very recently, Baek's group ${ }^{10}$ described a synthesis of CTFs at $400{ }^{\circ} \mathrm{C}$ through $\mathrm{P}_{2} \mathrm{O}_{5}$-mediated condensation of aromatic amide, which can be considered as a variation of ionothermal method of nitriles since $\mathrm{P}_{2} \mathrm{O}_{5}$ is known to catalyze the dehydration of amides into nitriles. Impressively, the approach that Jin, Tan and co-workers ${ }^{11}$ recently reported was by way of condensation of aldehyde and amidine under mild reaction conditions.

Herein, we are reporting a new synthetic strategy in which $\mathrm{C}-\mathrm{C}$ bonds in the CTFs were formed through aromatic nucleophilic substitution reactions under mild reaction condition (refluxing in toluene). To the best of our knowledge, this is the first time that aromatic nucleophilic substitution was used for $\mathrm{C}-\mathrm{C}$ bond formation in CTFs, and our method is different in many ways from the previously reported strategies. The assynthesized CTFs exhibited photocatalytic water splitting activity comparable to the CTFs made using previously reported methods.

While electrophilic substitution reactions are common for aromatic compounds, only some aryl halides can undergo substitution reactions with strong nucleophiles, ${ }^{12}$ and some of such reactions have been used in covalent organic frameworks (COFs) preparation. ${ }^{13}$ We therefore envisioned that the 
chlorines in cyanuric chloride could be displaced by a strong nucleophilic reagent, for example, phenyllithium, and such a reaction could be used to prepare CTFs. To test the feasibility of this idea, we carried out a model reaction of phenyllithium (PhLi) and cyanuric chloride, which, we delightly found, gave 2,4,6-triphenyl-1,3,5-triazine (TriPh-triazine) in an isolated yield of $87 \%$ (Scheme 1 and ESI $\dagger$ ).

Under very similar reaction conditions employed in the model reaction, two covalent triazine frameworks, CTF-1 and CTF-2 were successfully synthesized in gram-scale through aromatic nucleophilic substitution reaction of cyanuric chloride with para-dilithiumaromatic reagents in yields of $88 \%$ and $96 \%$, respectively. The para-dilithiumaromatic reagents (1,4-dilithiumbenzene and 4,4'-dilithiumbiphenyl) used in the CTFs preparation were obtained through reactions of para-diiodoaromatic reagents with $n$-butyllithium, a widely used reagent in organic synthesis (caution: a highly reactive reagent and standard operating procedure should be strictly followed) (Scheme 2 and ESI $\dagger$ ). Albeit aromatic nucleophilic substitution reactions of cyanuric chloride was previously employed in the preparation COFs through the formation of $\mathrm{C}-\mathrm{X}(\mathrm{X}=\mathrm{N}, \mathrm{S}, \mathrm{O})$ bonds,${ }^{14}$ such reactions have not been used to prepare COFs through $\mathrm{C}-\mathrm{C}$ bond formation.

CTF-1 and CTF-2 were characterized by Fourier transform infrared (FTIR) spectroscopy, solid-state ${ }^{13} \mathrm{C}$ cross polarization magic angle spinning nuclear magnetic resonance spectroscopy $\left({ }^{13} \mathrm{C}\right.$ CP/MAS NMR), X-ray photoelectron spectroscopy (XPS) analysis, thermogravimetric (TG) analysis, field emission scanning electron microscopy (FE-SEM), field emission transmission electron microscopy (FE-TEM), powder X-ray diffraction (PXRD) analysis, ultraviolet-visible diffuse reflectance spectroscopy (UV-Vis DRS) and solid-state photoemission spectroscopy.

The solid-state ${ }^{13} \mathrm{C}$ NMR spectra of CTF-1 and CTF-2 (Fig. 1A) showed the presence of $\mathrm{sp}^{2}$-hybridized carbon of the triazine unit $(\sim 170 \mathrm{ppm})$ as well as peaks of the aromatic rings (150-120 ppm). ${ }^{7 d}$ In the FTIR spectrum of CTF-1 (Fig. 1B) (CTF-2, see ESI, Fig. S12 $\dagger$ ), the disappearance of characteristic $\mathrm{C}-\mathrm{Cl}$ band of cyanuric chloride at $850 \mathrm{~cm}^{-1,},{ }^{8}$ together with the appearance of vibrational bands of triazine units at $1510 \mathrm{~cm}^{-1}$ (C-N stretching) and $1360 \mathrm{~cm}^{-1}$ (benzene ring breathing) implied the formation of CTFs. ${ }^{15}$

In the XPS spectra of the CTFs, the appearance of the peaks with binding energies of $286.2 \mathrm{eV}$ and $398.4 \mathrm{eV}$, assigned to $\mathrm{C} 1 \mathrm{~s}$ and $\mathrm{N}$ 1s of the triazine $(\mathrm{C}-\mathrm{N}=\mathrm{C})$ ring, respectively, together<smiles>Clc1nc(Cl)nc(Cl)n1</smiles>

cyanuric chloride

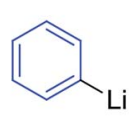

reflux

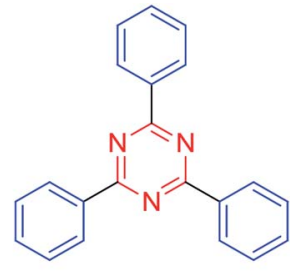

TriPh-triazine
Scheme 1 Reaction of phenyllithium and cyanuric chloride.

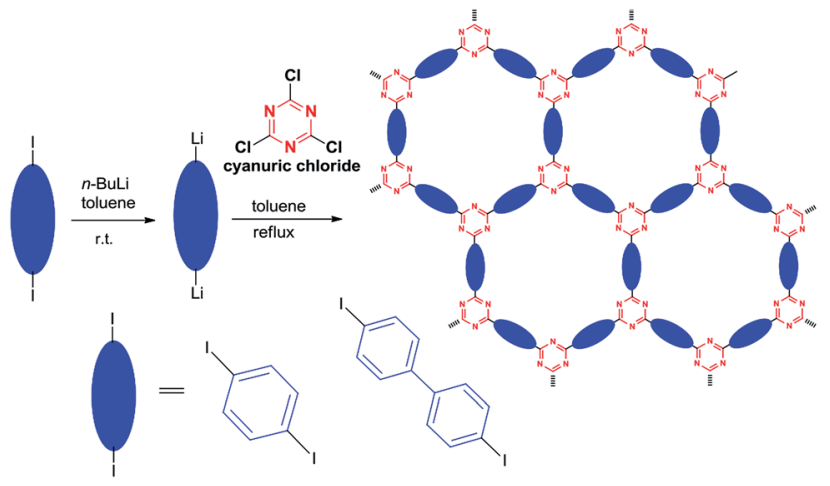

Scheme 2 Synthesis of CTF-1 and CTF-2.

with the peak at $\sim 284.3 \mathrm{eV}$, assigned to $\mathrm{C} 1 \mathrm{~s}$ of the aromatic carbons,${ }^{7 d, 16}$ implied the existence of the triazine and aromatic units. The ratios of $\mathrm{C}_{\text {triazine }}: \mathrm{C}_{\text {phenyl }}$ in the XPS spectra of CTF-1 and CTF-2 were $1: 2.55$ and $1: 5.71$, respectively, which were close to their theoretical values of $1: 3$ and $1: 6$ (Fig. 1C, S6 and S7 in ESI $\dagger$ ). The $\mathrm{O} 1$ s peaks seen in Fig. 1C are from physically adsorbed oxygen or water molecules which are usually found in carbon-based materials. ${ }^{9 b, 17}$ Scarcely any $\mathrm{Cl}$ or I peaks were found in the XPS spectra of CTF-1 and CTF-2 (Fig. 1C and Tables S1 and S2, ESI $\dagger$ ), which indicated the completeness of aromatic nucleophilic substitution of cyanuric chloride with the corresponding aryl dilithium reagents. TG analysis shows that both CTF-1 and CTF-2 started decomposition at $\sim 300{ }^{\circ} \mathrm{C}$ and experienced $\sim 60 \%$ weight loss at $1000{ }^{\circ} \mathrm{C}$ in nitrogen atmosphere
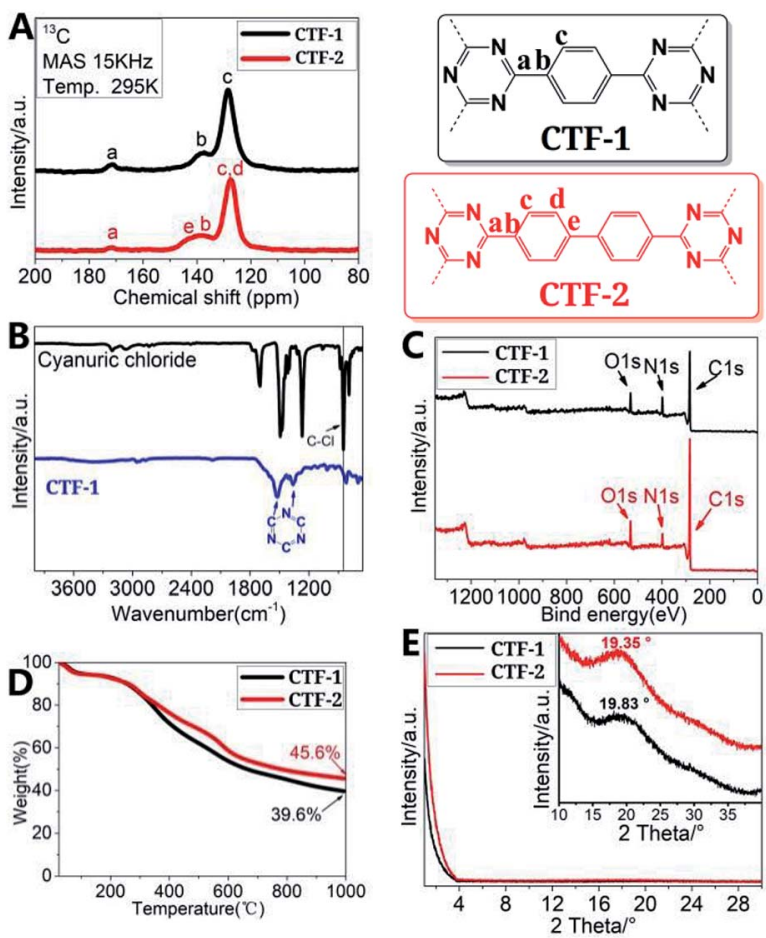

Fig. 1 (A) Solid-state ${ }^{13} \mathrm{C} C P / M A S N M R$ and repeating units of CTFs; (B) FTIR of CTF-1; (C) XPS survey spectra of CTFs; (D) TGA of CTFs; (E) PXRD of CTFS. 
(Fig. 1D). Extended porous network structures with branched morphologies for the as-synthesized CTFs were observed by using FE-SEM (Fig. S19, ESI $\dagger$ ) and TEM images (Fig. S19, ESI $\dagger$ ). Powder X-ray diffraction (PXRD) patterns of the CTFs showed no distinct sharp peaks, but curved broad peaks at around $2 \theta=$ $\sim 19-20^{\circ}$ (Fig. 1E) were observed, declaring their amorphous nature. No long-range crystallographic order for both CTFs indicated that the pore structures of the resulted CTFs maybe formed from unordered stacking and curling of the products. Such PXRD results are expected as the reaction was carried out under refluxing in toluene with vigorous stirring - the conditions unfavourable for the formation of crystalline products.

The porous properties of the both CTFs were evaluated by nitrogen adsorption-desorption measurement at $77 \mathrm{~K}$. Both samples exhibited the type IV adsorption isotherm character, ${ }^{18}$ typical for materials with mesoporous porosity (Fig. S8, ESI $\dagger$ ). The BET surface areas of CTF- 1 and CTF-2 are $c a .40-100 \mathrm{~m}^{2} \mathrm{~g}^{-1}$, and their pore diameters were calculated to be around 15$24 \mathrm{~nm}$ by Barrett-Joyner-Halenda (BJH) method (Table S3, ESI $\dagger$ ). The surface areas of the CTFs synthesized using our method are larger than those of the CTFs obtained though Brønsted-acid method $\left(5-8 \mathrm{~m}^{2} \mathrm{~g}^{-1}\right),{ }^{7 d}$ but smaller than that of the CTF-1 obtained through ionothermal $\left(\mathrm{ZnCl}_{2}\right)$ process $(791$ $\left.\mathrm{m}^{2} \mathrm{~g}^{-1}\right){ }^{7 \boldsymbol{b}}$ In the ionothermal $\left(\mathrm{ZnCl}_{2}\right)$ process, zinc chloride might act as template and present in the as-synthesized CTFs products, removing the zinc chloride from the as-synthesized CTFs products could potentially result in higher porosity. ${ }^{\mathbf{1 9}}$

The UV-Vis diffuse reflectance spectra (UV-Vis DRS) of CTF-1 and CTF-2 (Fig. S10, ESI $\dagger$ ) showed the intrinsic absorptions in the range of visible light, which are attributed to $\pi \rightarrow \pi^{*}$ electron transition of $\mathrm{C}_{\mathrm{sp}^{2}}$ and $\mathrm{N}_{\mathrm{sp}^{2}}$ in the polymeric networks. ${ }^{7 d}$ The Kubelka-Munk transformed reflectance spectra, ${ }^{20}$ as the mathematical equivalent transformation of UV-Vis DRS, showed band gaps of $2.62 \mathrm{eV}$ and $2.90 \mathrm{eV}$ of CTF- 1 and CTF-2 (Fig. 2A), respectively.

Theoretical calculation (DFT) indicated that the top levels of the valence band (VB) of the CTFs were more positive than the redox potential of $\mathrm{O}_{2} / \mathrm{H}_{2} \mathrm{O}(1.23 \mathrm{~V} v s$. NHE), while the bottom levels of their conduction band (CB) were more negative than the redox potential of $\mathrm{H}^{+} / \mathrm{H}_{2}(0 \mathrm{~V} v s$. NHE) (Fig. S9, ESI $\dagger)$, which implied that the CTFs could be used in photocatalytic water splitting, like those described in previous work. ${ }^{7 d, 21}$ The photocatalytic water splitting abilities (hydrogen evolution reaction, HER) of the as-synthesized CTFs were thus evaluated in
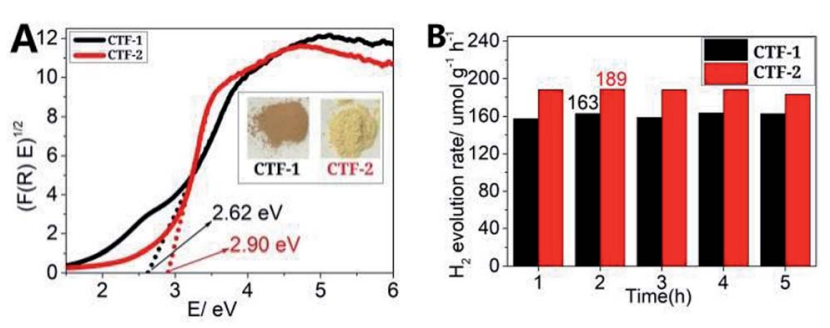

Fig. 2 (A) Kubelka-Munk transformed reflectance spectra of CTFs (inset: optical images of CTFs under visible light); (B) water-splitting reaction catalyzed by the two CTFs. comparison with those of previously reported results under visible light (>420 $\mathrm{nm}$ ) irradiation with triethanolamine (TEOA) as a photogenerated hole scavenger (ESI $\dagger$ ). Both CTFs showed water splitting ability under visible light (>420 nm), with CTF-2 possessing a slightly better catalytic activity than CTF-1 (Fig. 2B). Nonetheless, significant improvement was obviously needed on the photocatalytic activity toward water splitting for either CTFs.

In addition to the absorption property and photo-catalytic water splitting abilities (HER) of the as-synthesized CTFs, the emission property of the CTFs was also evaluated. Both CTFs exhibits similar fluorescence spectra under identical higherenergy irradiation (400 nm, violet), as shown in Fig. 3A and B.

Interestingly, CTF-2 was found to display distinct two-photoexcited fluorescence (TPF) under lower-energy irradiation using a femtosecond laser pulse (800 nm, infrared) (red curve in Fig. 3B). The observation of two-photon excited fluorescence property, or up-conversion phenomenon, of CTF-2 implied that CTF-2 has a higher two-photo absorption cross section. The difference in the two-photo absorption cross section between CTF-1 and CTF-2 could possibly be attributed to their structural difference. As described in a previous report, ${ }^{22}$ structural features including effectiveness of conjugation, increased conjugation length, good planarity and strong electron donating ability were all critical to enhance the two-photon absorption cross sections. While the aromatics-linked triazine frameworks in repetitive structures made the both CTFs maintain good planarity, the effectively conjugated aryl units are longer in the biphenyl-linked CTF-2. The molecular force field $(\mathrm{MM} 2)^{23}$ calculation (ESI $\dagger$ ) indicated that the charge distributions of triazine rings (electron deficient parts) in the two CTFs were almost the same $\left(\mathrm{C}_{\text {triazine }}{ }^{[+0.318]}\right)(\mathrm{ESI} \dagger)$, but the electron rich portions (aryl rings) of CTF-1 and CTF-2 hold different charge distributions (CTF-1 $\mathrm{C}_{\text {aromatic }}[-0.035]$ and CTF-2 $\left.\mathrm{C}_{\text {aromatic }}[-0.041]\right)$, which could partially explain why CTF-2 has a higher two-photo absorption cross section value. The up-conversion phenomenon of CTF-2 implies that CTF-2 could be excited to the same excited state by either absorbing one photo under higher-energy irradiation $(400 \mathrm{~nm})$ or absorbing two photons under lower-energy irradiation $(800 \mathrm{~nm})$. As lowerenergy irradiation $(800 \mathrm{~nm})$ provides a better penetration in scattering or absorbing media, ${ }^{24}$ especially in organic and biological materials, ${ }^{22}$ CTF-2 could possibly be used in nondestructive photoimaging and optical memory. ${ }^{25}$
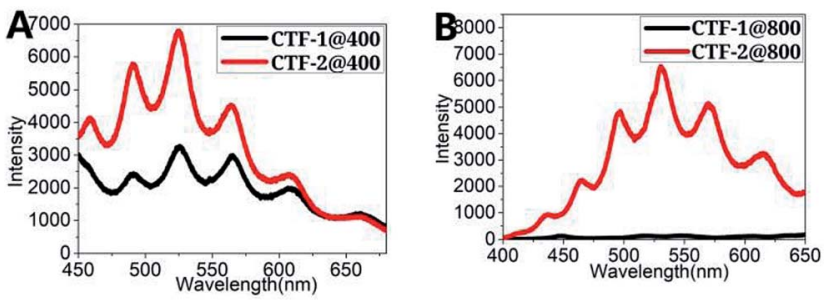

Fig. 3 (A) Solid-state single photo fluorescence spectra (A) $400 \mathrm{~nm}$ excitation) and two-photo fluorescence spectra (B) $(800 \mathrm{~nm}$ excitation) (the intensities of incident light on CTFs at $400 \mathrm{~nm}$ and $800 \mathrm{~nm}$ are the same, respectively). 


\section{Conclusions}

In conclusion, we have developed a novel synthetic approach to make covalent triazine-based frameworks CTF- 1 and CTF- 2 by means of aromatic nucleophilic substitution of cyanuric chloride with organic lithium reagents. The as-synthesized CTFs were found to exhibit the expected photocatalytic properties in water-splitting reaction (HER), similar to the CTFs obtained by other means. Additionally, CTF-2's two-photon fluorescence property could allow it to be a potentially useful material in nondestructive photoimaging and optical memory.

\section{Conflicts of interest}

The authors declare no conflict of interest.

\section{Acknowledgements}

This work was financially supported by the National Key Research Program (2017YFA0206500), the National Natural Science Foundation of China (21871281), the Science and Technology Commission of Shanghai Municipality (16DZ1100300, 18DZ1100403), and the Young Innovation Fund of Shanghai Advanced Research Institute (171008).

\section{Notes and references}

1 (a) F. J. Uribe-Romo, J. R. Hunt, H. Furukawa, C. Klock, M. O'Keeffe and O. M. Yaghi, J. Am. Chem. Soc., 2009, 131, 4570; (b) Z. H. Xiang and D. P. Cao, J. Mater. Chem. A, 2013, 1, 2691-2718; (c) A. G. Slater and A. I. Cooper, Science, 2015, 348, 6238; (d) P. Kuhn, A. Forget, D. S. Su, A. Thomas and M. Antonietti, J. Am. Chem. Soc., 2008, 130, 13333-13337; (e) J. X. Jiang, F. Su, A. Trewin, C. D. Wood, N. L. Campbell, H. Niu, C. Dickinson, A. Y. Ganin, M. J. Rosseinsky, Y. Z. Khimyak and A. I. Cooper, Angew. Chem., Int. Ed., 2007, 46, 8574-8578; (f) C. S. Diercks and O. M. Yaghi, Science, 2017, 355, 6328; $(g)$ A. I. Cooper, $A d v$. Mater., 2009, 21, 1291-1295; (h) N. Chaoui, M. Trunk, R. Dawson, J. Schmidt and A. Thomas, Chem. Soc. Rev., 2017, 46, 3302-3321; (i) R. Dawson, A. I. Cooper and D. J. Adams, Prog. Polym. Sci., 2012, 37, 530-563; (j) Y. H. Xu, S. B. Jin, H. Xu, A. Nagai and D. L. Jiang, Chem. Soc. Rev., 2013, 42, 8012-8031; (k) S. Das, P. Heasman, T. Ben and S. L. Qiu, Chem. Rev., 2017, 117, 1515-1563.

2 (a) P. J. Waller, F. Gandara and O. M. Yaghi, Acc. Chem. Res., 2015, 48, 3053-3063; (b) V. S. Vyas, F. Haase, L. Stegbauer, G. Savasci, F. Podjaski, C. Ochsenfeld and B. V. Lotsch, Nat. Commun., 2015, 6, 8508; (c) J. Artz, Chemcatchem, 2018, 10, 1753-1771; (d) A. V. Bavykina, M. G. Goesten, F. Kapteijn, M. Makkee and J. Gascon, Chemsuschem, 2015, 8, 809-812; (e) L. Chen, Y. Yang and D. L. Jiang, J. Am. Chem. Soc., 2010, 132, 9138-9143; (f) K. Iwase, T. Yoshioka, S. Nakanishi, K. Hashimoto and K. Kamiya, Angew. Chem., Int. Ed., 2015, 54, 11068-11072; (g) P. Katekomol, J. Roeser, M. Bojdys, J. Weber and A. Thomas, Chem. Mater., 2013, 25, 1542-1548; (h) P. Kaur, J. T. Hupp and S. T. Nguyen,
ACS Catal., 2011, 1, 819-835; (i) L. Z. Peng, P. Liu, Q. Q. Cheng, W. J. Hu, Y. A. Liu, J. S. Li, B. Jiang, X. S. Jia, H. Yang and K. Wen, Chem. Commun., 2018, 54, 44334436; (j) C. Perego and R. Millini, Chem. Soc. Rev., 2013, 42, 3956-3976; (k) X. R. Wang, X. Han, J. Zhang, X. W. Wu, Y. Liu and Y. Cui, J. Am. Chem. Soc., 2016, 138, 1233212335; (l) Y. G. Zhang and S. N. Riduan, Chem. Soc. Rev., 2012, 41, 2083-2094; $(m)$ K. Schwinghammer, S. Hung, M. B. Mesch, J. Senker and B. V. Lotsch, Energy Environ. Sci., 2015, 8, 3345-3353; (n) L. Li, W. Fang, P. Zhang, J. Bi, Y. He, J. Wang and W. Su, J. Mater. Chem. A, 2016, 4, 12402-12406; (o) J. Bi, W. Fang, L. Li, J. Wang, S. Liang, Y. He, M. Liu and L. Wu, Macromol. Rapid Commun., 2015, 36, 1799-1805.

3 (a) A. Bhunia, D. Esquivel, S. Dey, R. Fernandez-Teran, Y. Goto, S. Inagaki, P. Van der Voort and C. Janiak, J. Mater. Chem. A, 2016, 4, 13450-13457; (b) O. Buyukcakir, S. H. Je, S. N. Talapaneni, D. Kim and A. Coskun, ACS Appl. Mater. Interfaces, 2017, 9, 7209-7216; (c) S. S. Han, H. Furukawa, O. M. Yaghi and W. A. Goddard, J. Am. Chem. Soc., 2008, 130, 11580; (d) S. S. Han, J. L. Mendoza-Cortes and W. A. Goddard, Chem. Soc. Rev., 2009, 38, 1460-1476; (e) N. Huang, X. Chen, R. Krishna and D. L. Jiang, Angew. Chem., Int. Ed., 2015, 54, 2986-2990; (f) J. L. MendozaCortes, S. S. Han, H. Furukawa, O. M. Yaghi and W. A. Goddard, J. Phys. Chem. A, 2010, 114, 10824-10833; (g) J. L. Mendoza-Cortes, T. A. Pascal and W. A. Goddard, J. Phys. Chem. A, 2011, 115, 13852-13857; (h) P. Puthiaraj, S. S. Kim and W. S. Ahn, Chem. Eng. J., 2016, 283, 184-192; (i) K. K. Wang, H. L. Huang, D. H. Liu, C. Wang, J. P. Li and C. L. Zhong, Environ. Sci. Technol., 2016, 50, 48694876; (j) W. Zhang, C. Li, Y. P. Yuan, L. G. Qiu, A. J. Xie, Y. H. Shen and J. F. Zhu, J. Mater. Chem., 2010, 20, 64136415; (k) X. Zhu, C. C. Tian, S. M. Mahurin, S. H. Chai, C. M. Wang, S. Brown, G. M. Veith, H. M. Luo, H. L. Liu and S. Dai, J. Am. Chem. Soc., 2012, 134, 10478-10484.

4 (a) G. Das, B. P. Biswal, S. Kandambeth, V. Venkatesh, G. Kaur, M. Addicoat, T. Heine, S. Verma and R. Banerjee, Chem. Sci., 2015, 6, 3931-3939; (b) S. Y. Ding, M. Dong, Y. W. Wang, Y. T. Chen, H. Z. Wang, C. Y. Su and W. Wang, J. Am. Chem. Soc., 2016, 138, 3031-3037; (c) Z. P. Li, Y. W. Zhang, H. Xia, Y. Mu and X. M. Liu, Chem. Commun., 2016, 52, 6613-6616; (d) L. Xiang, Y. L. Zhu, S. Gu, D. Y. Chen, X. Fu, Y. D. Zhang, G. P. Yu, C. Y. Pan and Y. H. Hu, Macromol. Rapid Commun., 2015, 36, 15661571.

5 (a) A. Rengaraj, P. Puthiaraj, Y. Haldorai, N. S. Heo, S. K. Hwang, Y. K. Han, S. Kwon, W. S. Ahn and Y. S. Huh, ACS Appl. Mater. Interfaces, 2016, 8, 8947-8955; (b) H. R. Wang, W. W. Zhu, L. Z. Feng, Q. Chen, Y. Chao, Z. L. Dong and Z. Liu, Nano Res., 2018, 11, 3244-3257.

6 (a) C. R. Mulzer, L. X. Shen, R. P. Bisbey, J. R. McKone, N. Zhang, H. D. Abruna and W. R. Dichtel, ACS Cent. Sci., 2016, 2, 667-673; (b) F. X. Yang, S. S. Cheng, X. T. Zhang, X. C. Ren, R. J. Li, H. L. Dong and W. P. Hu, Adv. Mater., 2018, 30, 1702415; (c) X. Feng, X. S. Ding and D. L. Jiang, Chem. Soc. Rev., 2012, 41, 6010-6022. 
7 (a) M. J. Bojdys, J. Jeromenok, A. Thomas and M. Antonietti, Adv. Mater., 2010, 22, 2202; (b) P. Kuhn, M. Antonietti and A. Thomas, Angew. Chem., Int. Ed., 2008, 47, 3450-3453; (c) P. Kuhn, A. Thomas and M. Antonietti, Macromolecules, 2009, 42, 319-326; (d) Z. A. Lan, Y. X. Fang, Y. F. Zhang and X. C. Wang, Angew. Chem., Int. Ed., 2018, 57, 470-474.

8 (a) S. Dey, A. Bhunia, D. Esquivel and C. Janiak, J. Mater. Chem. A, 2016, 4, 6259-6263; (b) P. Puthiaraj, S. M. Cho, Y. R. Lee and W. S. Ahn, J. Mater. Chem. A, 2015, 3, 67926797.

9 (a) Z. H. Xiang and D. P. Cao, Macromol. Rapid Commun., 2012, 33, 1184-1190; (b) Z. H. Xiang, D. P. Cao, L. Huang, J. L. Shui, M. Wang and L. M. Dai, Adv. Mater., 2014, 26, 3315; (c) Z. H. Xiang, X. Zhou, C. H. Zhou, S. Zhong, X. He, C. P. Qin and D. P. Cao, J. Mater. Chem., 2012, 22, 2266322669.

10 S. Y. Yu, J. Mahmood, H. J. Noh, J. M. Seo, S. M. Jung, S. H. Shin, Y. K. Im, I. Y. Jeon and J. B. Baek, Angew. Chem., Int. Ed., 2018, 57, 8438-8442.

11 (a) M. Y. Liu, Q. Huang, S. L. Wang, Z. Y. Li, B. Y. Li, S. B. Jin and B. Tan, Angew. Chem., Int. Ed., 2018, 57, 11968-11972; (b) K. W. Wang, L. M. Yang, X. Wang, L. P. Guo, G. Cheng, C. Zhang, S. B. Jin, B. Tan and A. Cooper, Angew. Chem., Int. Ed., 2017, 56, 14149-14153.

12 (a) D. P. Green and D. Zuev, in Encyclopedia of Reagents for Organic Synthesis, John Wiley \& Sons, Ltd, 2008, DOI: 10.1002/047084289X.rp076.pub2; (b) L. Xu, P.-F. Wang, J.-J. Zhang, W. Wu, J.-W. Shi, J.-F. Yuan, H. Han and H. Wang, RSC Adv., 2015, 5, 51745-51749.

13 B. Zhang, M. F. Wei, H. Y. Mao, X. K. Pei, S. A. Alshmimri, J. A. Reimer and O. M. Yaghi, J. Am. Chem. Soc., 2018, 140, 12715-12719.
14 P. Puthiaraj, Y. R. Lee, S. Q. Zhang and W. S. Ahn, J. Mater. Chem. A, 2016, 4, 16288-16311.

15 Y. H. Xiong, Y. M. Qin, L. J. Su and F. G. Ye, Chem. - Eur. J., 2017, 23, 11037-11045.

16 Z. H. Sheng, L. Shao, J. J. Chen, W. J. Bao, F. B. Wang and X. H. Xia, ACS Nano, 2011, 5, 4350-4358.

17 (a) D. C. Wei, Y. Q. Liu, Y. Wang, H. L. Zhang, L. P. Huang and G. Yu, Nano Lett., 2009, 9, 1752-1758; (b) L. T. Qu, Y. Liu, J. B. Baek and L. M. Dai, ACS Nano, 2010, 4, 13211326.

18 K. A. Cychosz, R. Guillet-Nicolas, J. Garcia-Martinez and M. Thommes, Chem. Soc. Rev., 2017, 46, 389-414.

19 S. J. Ren, M. J. Bojdys, R. Dawson, A. Laybourn, Y. Z. Khimyak, D. J. Adams and A. I. Cooper, Adv. Mater., 2012, 24, 2357-2361.

20 M. Otsuka, Powder Technol., 2004, 141, 244-250.

21 (a) A. Fujishima and K. Honda, Nature, 1972, 238, 37; (b) A. Kudo and Y. Miseki, Chem. Soc. Rev., 2009, 38, 253-278; (c) G. Zhang, Z.-A. Lan and X. Wang, Angew. Chem., Int. $E d .$, 2016, 55, 15712-15727.

22 B. A. Reinhardt, L. L. Brott, S. J. Clarson, A. G. Dillard, J. C. Bhatt, R. Kannan, L. X. Yuan, G. S. He and P. N. Prasad, Chem. Mater., 1998, 10, 1863-1874.

23 N. L. Allinger, J. Am. Chem. Soc., 1977, 99, 8127-8134.

24 M. Albota, D. Beljonne, J. L. Bredas, J. E. Ehrlich, J. Y. Fu, A. A. Heikal, S. E. Hess, T. Kogej, M. D. Levin, S. R. Marder, D. McCord-Maughon, J. W. Perry, H. Rockel, M. Rumi, C. Subramaniam, W. W. Webb, X. L. Wu and C. Xu, Science, 1998, 281, 1653-1656.

25 (a) H. M. Kim and B. R. Cho, Chem. Rev., 2015, 115, 50145055; (b) Q. Liu, B. D. Guo, Z. Y. Rao, B. H. Zhang and J. R. Gong, Nano Lett., 2013, 13, 2436-2441. 